\title{
KBV will bundeseinheitliche Vergütung
}

— Nach der Auffassung der KBV bestehen nach der Vergütungsreform zum 1.1.2009 Streubreiten von bis zu $100 \%$. KVen wie Westfalen-Lippe und Nordrhein hätten z.B. mit unterdurchschnittlichen Honorarzuwächsen und geringen Regelleistungsvolumina (RLV) zu kämpfen. Im Durchschnitt betrage die Vergütung je Versicherten bundesweit 172 Euro. In Westfalen-Lippe seien es nur 152 Euro, in Nordrhein 155 Euro. Diese großen Unterschiede zwischen den Regionen sind nach Auffassung der KBV historisch gewachsen. Durch die Honorarreform hätte sich hier nichts geändert. Langfristig sei eine einheitliche Vergütung nur dann zu verwirklichen, wenn sich die KVen von der re- gionalen Honorarverteilungshoheit verabschieden würden. Die Unterschiede zwischen den RLV-Fallwerten könnten nur beseitigt werden, wenn auch dieser letzte Schritt vollzogen werde.

\begin{tabular}{|c|c|c|c|}
\hline \multirow[t]{2}{*}{$\begin{array}{l}\text { Kassenärztliche } \\
\text { Vereinigung }\end{array}$} & \multirow{2}{*}{$\begin{array}{l}\text { Simulierte Gesamt- } \\
\text { vergütung } 2009 \\
\text { in Mio. Euro }\end{array}$} & \multicolumn{2}{|c|}{$\begin{array}{l}\text { Veränderung Gesamthonorar } \\
\text { gegenüber } 2007\end{array}$} \\
\hline & & in Mio. Euro & prozentual \\
\hline 1 & 2 & 3 & 4 \\
\hline $\begin{array}{l}\text { Schleswig- } \\
\text { Holstein }\end{array}$ & 943,5 & 35,1 & $3,9 \%$ \\
\hline Hamburg & 676,3 & 45,9 & $7,3 \%$ \\
\hline Bremen & 312,1 & 22,4 & $7,7 \%$ \\
\hline Niedersachsen & $2.882,4$ & 358,3 & $14,2 \%$ \\
\hline Westfalen-Lippe & $3.259,1$ & 262,4 & $8,8 \%$ \\
\hline Nordrhein & $3.131,1$ & 109,6 & $3,6 \%$ \\
\hline Hessen & $2.207,1$ & 191,6 & $9,5 \%$ \\
\hline Rheinland-Pfalz & $1.329,2$ & 72,2 & $5,7 \%$ \\
\hline $\begin{array}{l}\text { Baden- } \\
\text { Württemberg }\end{array}$ & $3.747,0$ & 53,7 & $1,5 \%$ \\
\hline Bayern & $4.924,6$ & 312,5 & $6,8 \%$ \\
\hline Berlin & $1.285,5$ & 124,2 & $10,7 \%$ \\
\hline Saarland & 357,2 & 36,4 & $11,3 \%$ \\
\hline $\begin{array}{l}\text { Mecklenburg- } \\
\text { Vorpommern }\end{array}$ & 669,7 & 99,5 & $17,5 \%$ \\
\hline Brandenburg & 861,3 & 104,7 & $13,8 \%$ \\
\hline Sachsen-Anhalt & 832,4 & 122,2 & $17,2 \%$ \\
\hline Thüringen & 765,7 & 136,2 & $21,6 \%$ \\
\hline Sachsen & $1.496,9$ & 216,3 & $16,9 \%$ \\
\hline Gesamt & $29.681,1$ & $2.303,3$ & $8,4 \%$ \\
\hline West & $25.055,1$ & $1.624,3$ & $6,9 \%$ \\
\hline Ost & 4626,0 & 679,0 & $17,2 \%$ \\
\hline
\end{tabular}

\section{MMW Kommentar}

Die Einschätzung der durch ihren Vorsitzenden geäußerten Ursachen des Scheiterns der Honorarreform 2009 durch die $K B V$ ist bemerkenswert und gefährlich zugleich. Als Ursache der Fehlverteilungen wird der Gesetzgeber genannt, obgleich die Fehler nachweislich von der Selbstverwaltung gemacht wurden.

Zwar räumt der KBV-Vorsitzende ein, mit der Steigerung der Leistungen im Vorwegabzug zulasten der RLV einen Aspekt der Honorarreform falsch eingeschätzt zu haben. Angesichts der Tatsache, dass diese Vorwegabzüge allerdings bereits mit der Reform zum 1.4.2005 vorgesehen waren und in den wenigen KVen, die dies $z u$ diesem Zeitpunkt bereits umgesetzt hatten, genau zu diesem Desaster führten, das nach dem 1.1.2009 zu beobachten war, ist diese Ausrede nicht zulässig. Die nun geplante Änderung, dass künftig erst die RLV mit Honorar bedient werden und dann die freien Leistungen, hätte von Anfang an eingeführt werden müssen und kommt zu spät. Die mittlerweile eingefahrenen Bahnen werden zerstört und neue Verwerfungen sind absehbar. So wird es keine Ruhe unter der Vertragsärzteschaft geben. Die Schuld liegt bei zentralen Regelungen, die regionalen Besonderheiten nicht gerecht werden. Zentralismus kann niemals die Lösung für die momentane bundesweite Honorarmisere sein. Nötig ist eine $A b$ kehr von diesem eindeutig falschen Weg!

\section{Bleibeprämien sind unzulässig}

- Krankenkassen, die Zusatzbeiträge erheben, dürfen Mitglieder nicht mit Prämien zum Bleiben bewegen. Die Koppelung von Halteprämien und Zusatzbeiträgen ist nach der Auffassung des Bundesversicherungsamtes (BVA) unzulässig. Eine Krankenkasse sei zur Erhebung eines Zusatzbeitrages gesetzlich verpflichtet, wenn sie ihren Finanzbedarf durch die Zuweisungen aus dem Gesundheitsfonds nicht decken kann. Die Mitglieder der Krankenkasse hätten in diesem Fall das Recht, bis zur erstmaligen Fälligkeit des Zusatzbeitrages, die Mitgliedschaft in der Krankenkasse zu kündigen. Wenn eine Krankenkasse versucht, ihre Mitglieder durch Zahlung einer Halteprämie von der Ausübung ihres Kündigungsrechtes abzuhalten, werde der Eindruck erweckt, es seien ausreichende finanzielle Mittel vorhanden und damit die Notwendigkeit des Zusatzbeitrages infrage gestellt.

\section{MMW Kommentar}

Auch wenn sich die Stellungnahme des BVA sich auf Zahlungsangebote von Kassen unmittelbar im Zusammenhang mit der Ankündigung einer Kündigung der Mitgliedschaft eines Versicherten bezieht, kann in diesem Zusammenhang auch auf das gesamte Bonuswesen der Kassen reflektiert werden. Es versteht sich von selbst, dass eine Kasse, die mit ihrem Beitragsgeld nicht auskommt, nicht auch noch mit Prämien für z.B. die regelmäßige Teilnahme an Gesundheitsförderungsmaßnahmen werben darf. Abgesehen davon, dass es Aufgabe und Interessenlage des Arztes sein muss, seine Patienten zu solchen Maßnahmen zu motivieren, erübrigt und erledigt sich damit - zumindest für Versicherte solcher Kassen - der angeblich kostenlose Eintrag in ein sog. Bonusheft. 